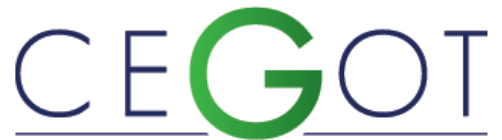

Centro de Estudos de Geografia e Ordenamento do Território
Geografia e Ordenamento do Território, Revista Eletrónica Centro de Estudos de Geografia e Ordenamento do Território http://cegot.org

Muñoz-Vera, Francisco

Universidad Católica de Temuco, Facultad de Recursos

Naturales, Laboratorio de Planificación Territorial

4780000, Rudecindo Ortega 02950, Temuco, Chile

francisco.munoz@uct.cl

ROJAS-HERNÁNDEZ, JORGE

Universidad de Concepción, Facultad de Ciencias Sociales,

Departamento de Sociología

4030000, Barrio Universitario S/N, Concepción, Chile

jrojas@udec.cl

\title{
Vulnerabilidad social en hogares desplazados por el terremoto y tsunami del año 2010 en la localidad de Dichato, Región del Bío-Bío, Chile \\ Vulnerabilidade social nas famílias deslocadas pelo terremoto e tsunami de 2010 na aldeia de Dichato, Região de Bío-Bío, Chile \\ Social vulnerability in households displaced by the 2010 earthquake and tsunami in the village of Dichato, Bío-Bío Region, Chile
}

Referência: Muñoz-Vera, Francisco; Rojas-Hernández, Jorge (2018). Vulnerabilidad social en hogares desplazados por el terremoto y tsunami del año 2010 en la localidad de Dichato, Región del Bío-Bío, Chile. Revista de Geografia e Ordenamento do Território (GOT), n. ${ }^{\circ} 13$ (junho). Centro de Estudos de Geografia e Ordenamento do Território, p. 289-314, dx.doi.org/10.17127/got/2018.13.013

\section{RESUMEN}

Este artículo describe los niveles de vulnerabilidad social de hogares desplazados por el terremoto y tsunami del año 2010 en la localidad de Dichato. Para los hogares afectados, los activos de vulnerabilidad (recursos materiales, económicos y sociales que disponen) adquieren un rol fundamental para enfrentar condiciones profundamente alteradas por un desastre natural. Desde un enfoque multimétodo se identifican activos y estrategias utilizadas por los hogares para movilizar dichos recursos durante la estadía en un campamento de emergencia. Asimismo, se elabora un índice a través de un análisis factorial de correspondencias, que permitió identificar factores latentes de vulnerabilidad denominados: Condicionantes de vulnerabilidad y Mecanismos de adaptación. Estos factores describen limitaciones y capacidades de los hogares para enfrentar un contexto de alta complejidad social.

Palabras clave: Vulnerabilidad social, activos de vulnerabilidad, desplazados, terremoto, tsunami.

\section{RESUMO}

Este artigo descreve os níveis de vulnerabilidade social das famílias deslocadas pelo terremoto de 2010 e o tsunami na cidade de Dichato. Para os agregados familiares afetados, os recursos vulneráveis (recursos materiais, econômicos e sociais disponíveis) adquirem um papel fundamental para enfrentar condições profundamente alteradas por uma catástrofe natural. Uma abordagem multi-método identifica ativos e estratégias usados pelas famílias 
para mobilizar esses recursos durante sua permanência em um campo de emergência. Do mesmo modo, um índice é elaborado através de uma análise de correspondência fatorial, que permitiu identificar fatores de vulnerabilidade latente denominados: Restrições de vulnerabilidade e Mecanismos de adaptação. Esses fatores descrevem limitações e capacidades das famílias para enfrentar um contexto de alta complexidade social.

Palavras-chave: Vulnerabilidade social, vulnerabilidade ativa, deslocado, terremoto, tsunami.

\section{ABSTRACT}

This article describes the levels of social vulnerability in households displaced in the village of Dichato after the 2010 earthquake and tsunami. Vulnerability assets (i.e., physical, economic and social resources available to individuals) play a fundamental role in facing conditions that have been deeply disrupted by natural disasters. A multimethod approach identifies the assets and strategies households applied to mobilize such resources during an emergency camp stay. An index was developed, through a factorial correspondence analysis, to identify underlying vulnerability factors, namely, vulnerability constraints and adaptation mechanisms. These factors describe both restrictions and capabilities of families to face a highly social complex context.

Keywords: Social vulnerability, vulnerability assets, displaced, eartquake, tsunami.

\section{Introducción}

Este trabajo tiene como objetivo determinar niveles de vulnerabilidad social en hogares desplazados a causa del terremoto y tsunami del 27 de febrero de 2010 a través de la construcción de un índice y la descripción de estrategias de movilización de activos. Para dar cumplimiento a dicho objetivo, se identificaron los principales activos disponibles en hogares desplazados, se describen las estrategias de movilización de tales recursos, para posteriormente proponer un índice en base a la posesión de activos que permite conocer el nivel de vulnerabilidad y los activos que adquieren mayor relevancia para los hogares desplazados.

El 27 de febrero de 2010 la zona central de Chile fue afectada por un fuerte sismo de magnitud $8.8^{\circ} \mathrm{Mw}$ según los registros del Servicio Geológico de Estados Unidos (USGS), cuyo epicentro se ubicó en las costas de la Región del Maule, específicamente a 43 km. al sur de la localidad de Cobquecura. Este movimiento afectó un tramo de 700 kilómetros entre las 
regiones de Valparaíso y La Araucanía, territorio que concentra aproximadamente el $80 \%$ de la población del país (CEPAL, 2010).

El primer reporte de la CEPAL sobre el terremoto de 2010, identificó al borde costero, y particularmente a algunas zonas donde el turismo y la pesca artesanal son la principal actividad económica, como las localidades doblemente afectadas tanto por el movimiento sísmico, como por el posterior tsunami. Específicamente la localidad de Dichato fue la más destruida en la región del Bío-Bío (Ibíd., p.43). Esta bahía se ubica a $10 \mathrm{~km}$. al norte de Tomé, capital comunal del mismo nombre, posee 3.869 habitantes según estimaciones oficiales (INE, 2010) y es particularmente conocida por su vocación turística asociada a su balneario y gastronomía.

La destrucción de esta localidad fue tal, que obligó a reasentar a parte importante de su población en viviendas de emergencia bajo complejas condiciones de vida, cuestión que motivó a conocer cómo los hogares enfrentaron un contexto post-desastre, y particularmente cómo abordaron sus condiciones de vulnerabilidad frente a un evento de las características antes señaladas.

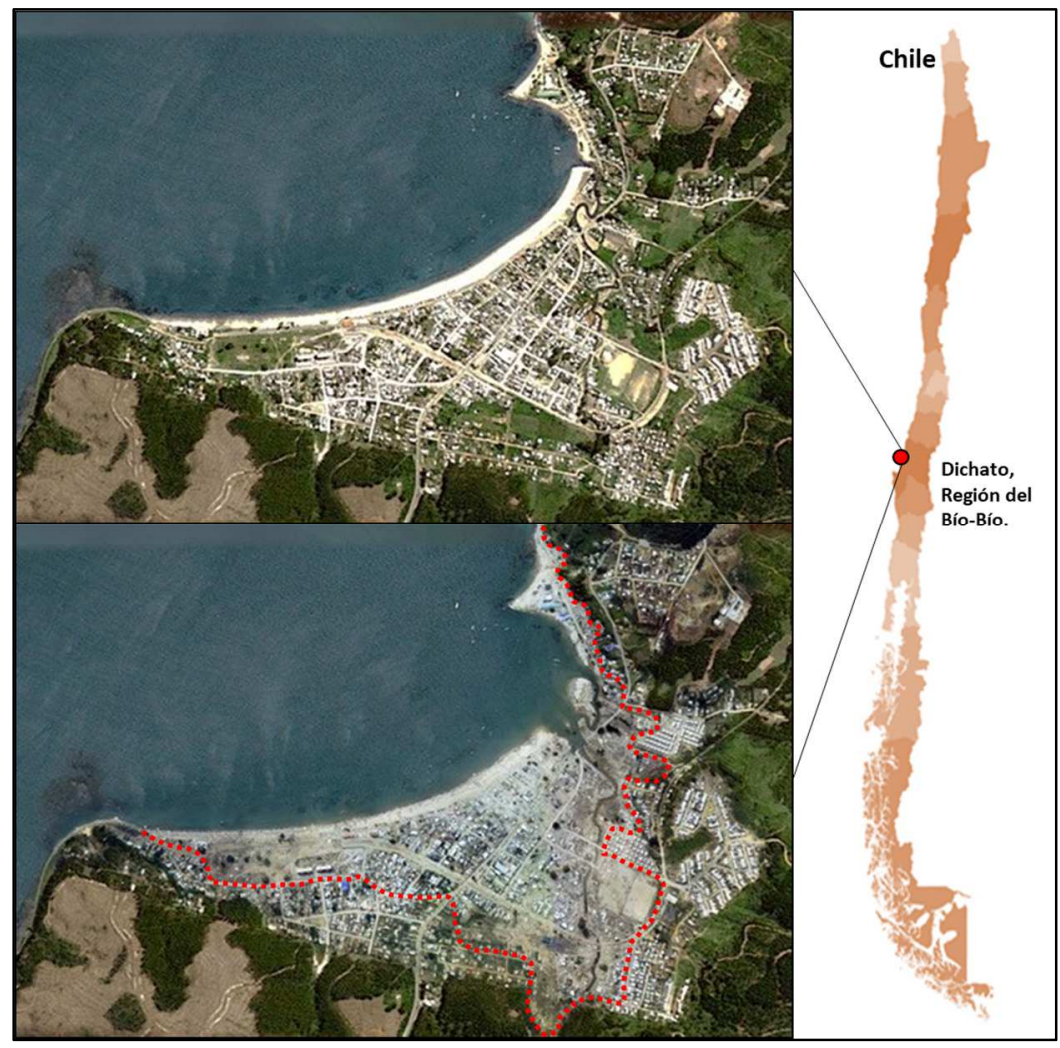

Figura 1 - Localidad de Dichato antes y después del terremoto-tsunami de 2010 (Fuente: Elaboración propia con imágenes capturadas por Google Earth). 
La Figura 1 en la imagen superior muestra la localidad de Dichato en una captura de Google Earth del 24 de enero de 2010. Este asentamiento concentraba parte importante de su población en el borde costero con acceso y exposición directa al mar. La imagen inferior corresponde al día 03 de abril de 2010, después de un mes de ocurrido el terremoto y tsunami; en ella se puede apreciar el alcance de la destrucción del mar que como referencia ha sido delimitado por una línea punteada.

En un contexto de destrucción y posterior desarrollo de un proceso de reasentamiento de los hogares, son los activos de vulnerabilidad -entendidos como el subconjunto de recursos, tanto materiales como simbólicos, que permiten a los individuos desarrollarse en la sociedad (Kaztman, 1999)- los que adquieren un rol central para que hogares en situación de alta complejidad social puedan enfrenar dicho contexto (Blaikie et al., 1996).

Es necesario señalar que un desastre natural, que implica destrucción del lugar de origen, pérdidas de recursos materiales, desarticulación del tejido social e incertidumbre respecto de las modificaciones que puedan existir también en los recursos simbólicos, justifican conocer cómo se reestructura la disponibilidad de dichos recursos y cómo las personas afectadas movilizan tales activos para enfrentar su situación de vulneración en un contexto post-desastre.

\section{El debate en torno a la vulnerabilidad y los desplazados}

\subsection{El contexto vulnerabilidad social}

Si bien se sabe que ante el desencadenamiento de un desastre los niveles de vulnerabilidad social se incrementan, es importante comprender cómo los hogares enfrentan este tipo de situaciones. Trabajos como los de Álvarez y Tuñón (2016), Sandoval-Ayala y Soares-Moraes (2015), Araújo y Rosa (2014), Cutter et al. (2003), Cutter y Finch (2008), entre otros, dan cuenta de la importancia de los activos que disponen los hogares para enfrentar escenarios de alta vulnerabilidad social frente a riesgos y desastres naturales.

La vulnerabilidad es uno de los conceptos que cobra particular importancia y que es realzado tanto por la literatura especializada, como por los organismos internacionales a 
cargo de enfrentar situaciones de desastre. Durante la última década los aportes se han hecho desde distintas disciplinas, permitiendo ampliar la utilización del marco de la vulnerabilidad mucho más allá del fenómeno de la pobreza. Sin embargo, la vulnerabilidad social busca también identificar la sensibilidad de la población para responder y recuperarse ante los impactos de un desastre, resultando como constructo sumamente complejo y que no es posible capturar con una sola variable (Cutter y Finch, 2008).

Uno de los primeros investigadores que posicionó la noción de vulnerabilidad es Moser $(1996,1998)$ a través de lo que denominó "asset/vulnerability framework". Posteriormente será Kaztman $(1999,2001)$ quien reconoce la debilidad de los hogares pobres para desenvolverse en su vida cotidiana cuando se desencadenan crisis económicas, pero que serían capaces de enfrentar con una adecuada "gestión" de sus propios activos. Tanto Moser como Kaztman, destacan la importancia de centrar el análisis en cómo se puede ver modificada la oferta de oportunidades en el marco de una integración al bienestar social, particularmente en sus recursos y activos que poseen los hogares para acceder a ella. Sin duda, este tipo de relación es cambiante y dinámica, de ahí su complejidad, particularmente cuando se asocia al riesgo de permanecer o caer en situación de pobreza, privación social o ante un desastre natural (Wormald et al., 2002; citado en Kaztman y Wormald, 2002).

Barrenechea et al. (2000) se refieren a la vulnerabilidad como una capacidad diferenciada de enfrentar las condiciones, principalmente económicas, ante un desastre y que, por extensión, involucra ciertos niveles de organización e institucionalización de planes de mitigación, preparación, prevención y recuperación; por lo que enfrentar un estado de vulneración se encuentra fuertemente ligado al ámbito del desarrollo.

Desde esta perspectiva resulta importante considerar la noción de vulnerabilidad en el marco de las implicancias sociales que conlleva. Tal como se indicó, el abordaje tradicional de la vulnerabilidad social se encuentra vinculado a las dimensiones de la pobreza, permitiendo abordar este fenómeno desde otros enfoques. Por ejemplo, para Filgueira y Peri (2004) no es posible asociar ni utilizar como sinónimo de vulnerabilidad la idea de movilidad descendente. Lo anterior estaría determinado por ser esta -la movilidad descendente- una consecuencia probable de la condición de vulnerable, por lo que la vulnerabilidad como concepto alude más bien a la situación de riesgo relativo frente a un acontecimiento que puede alterar el bienestar de las personas (Golovanevsky, 2007). 
Los estudios de la vulnerabilidad y las perspectivas hasta aquí señaladas, se han influenciado fuertemente por dos principales propuestas que buscan la integración de los activos a la discusión sobre la vulnerabilidad social.

La primera de ellas, tal como se indicó, abordada en Moser (1996; 1998) y Moser et al. (1997), se concentró en mejorar programas destinados a la reducción de la pobreza a través de su análisis de asset-vulnerability framework o marco de activos-vulnerabilidad. Su análisis buscaba conocer las respuestas que pueden dar los hogares pobres, particularmente cuando se enfrentan a situaciones de crisis. De esta manera, no se concentran en una perspectiva tradicional referente a los recursos de los que los pobres carecen, sino en cómo son movilizados los recursos o activos disponibles (Filgueira y Peri 2004).

La segunda propuesta alude a la aproximación de estructura de oportunidades - activos vulnerabilidad. Desde esta perspectiva, la vulnerabilidad puede ser abordada como una relación que se expresa mejor entre dos términos: por un lado los activos a disposición del hogar y su capacidad de movilización (expresadas como atributos individuales compartidos o atributos colectivos de los hogares) y, por otro, la estructura de oportunidades de la sociedad, que se refiere a las probabilidades de acceso a bienes o servicios que tienen los hogares y que inciden en su bienestar (Filgueira y Peri 2004).

Será particularmente la perspectiva de Filgueira (2001) y Filgueira y Peri (2004) donde el concepto de vulnerabilidad se enmarca en el enfoque de activos de vulnerabilidad, la que permitirá comprender la vulnerabilidad social como un conjunto de las dos propuestas antes señaladas. La primera de ellas, responderá a una dimensión macro y es relativo a la estructura de oportunidades vigente en un determinado lugar; mientras que la segunda se define en una dimensión micro y refiere a los activos con los que cuentan las personas $u$ hogares.

\subsection{Disponibilidad y movilización de activos}

La vulnerabilidad es una condición producto de desigualdades que sin duda influyen y dan forma a susceptibilidades y capacidades de respuestas ante desastres naturales (Cutter, et al., 2003), donde dicha capacidad de respuesta estará directamente relacionada con el nivel de vulnerabilidad existente en hogares desplazados. 
La vulnerabilidad puede ser abordada como un concepto relacional, que se expresa principalmente en función de la disponibilidad de activos en el hogar, la capacidad para movilizar tales recursos y en reconocer la estructura de oportunidades que ofrece el entorno. Respecto de la disponibilidad podemos señalar que alude a la posesión, control y manejo de instrumentos materiales y simbólicos para el desempeño del individuo en la sociedad (Filgueira y Peri, 2004). De este modo, la experiencia laboral, el desarrollo de ciertas habilidades, el nivel educativo, las destrezas y capacidades para comprender diferentes esferas de la sociedad, la pertenencia a redes de información e influencia, todos ellos favorecen la capacidad ascendente de las personas y les permiten estar más y mejor preparados ante situaciones de riesgo.

Asimismo, se añaden elementos propios del capital cultural como el manejo de algunos códigos comunicacionales, participación o la existencia de ciertos recursos adscritos por parte de la familia, etapa del ciclo de vida, número de hijos, capacidad de acumulación u ahorro familiar, entre otros. Todos estos atributos son llamados "activos" en analogía al análisis económico referente a los recursos con los que se cuenta (Filgueira y Peri, 2004). Estos elementos cobrarán particular importancia en la capacidad que tienen los hogares para enfrentar un contexto vulnerable. Es así que las herramientas o insumos que se ocupan y movilizan ante un escenario con cierto nivel de incertidumbre, resultan centrales desde esta perspectiva de análisis.

Para Pizarro (2001), los recursos que disponen las familias se encuentran vinculados a diferentes dimensiones de la vida social, siendo posible clasificar en: trabajo, capital humano, capital físico y relaciones sociales. De esta manera, la capacidad de movilización de recursos para enfrentar un contexto de vulnerabilidad se daría en dicho ámbito.

Ahora bien, para Kaztman (2000) los activos se pueden clasificar en base a tres tipos principales; activos físicos: entendidos como bienes materiales entre los que se encontrarían las viviendas, terrenos, vehículos o medios de transporte propios; activos financieros: estos refieren al capital que ofrece liquidez y que determina la posibilidad de acceder a créditos; y activos de capital humano: corresponden principalmente al estado de salud, nivel de educación, calificación, destrezas y habilidades. 
En cambio para Moser (1998) existen al menos cinco categorías de recursos para enfrentar un contexto vulnerable y así satisfacer las necesidades básicas: trabajo, considerado como la categoría más importante; capital humano, referido principalmente al estado de salud que determina la capacidad de trabajar, educación y habilidades; recursos productivos, que en el caso de hogares urbanos correspondería a la vivienda; relaciones del hogar, centradas en los mecanismos para incrementar recursos y restringir consumos; y capital social, correspondiente a las redes de apoyo entre las familias y la comunidad. Como es posible apreciar, no existe una estructura única en función de las dimensiones que componen los activos, pero si hay un relativo consenso y algunos elementos transversales que permitirían definir una batería común o portafolio de activos posibles de identificar en los hogares.

Resultará prioritario comprender que los activos no pueden ser considerados simplemente como un stock de recursos que disponen los hogares, sino que su existencia estaría directamente relacionada con la capacidad de los hogares para movilizarlos. En este sentido, Busso (2006) plantea que, tanto los recursos disponibles, como las estrategias desarrolladas durante su movilización, es lo que condicionaría la capacidad y forma de respuesta de los hogares ante un cambio brusco del entorno. Por lo tanto, cuando un hogar se enfrenta a las consecuencias de un desastre natural, en este caso un terremoto y posterior tsunami, los escasos recursos con los que queda, la capacidad de uso y movilización de estos, serán centrales para enfrentar un contexto de alta complejidad.

\subsection{La discusión en torno a los desplazados ambientales}

Una de las principales características de los desastres naturales, y que los define por excelencia, corresponde a la incertidumbre que generan con su desencadenamiento. Esta se traduce principalmente en la complejidad, e incluso imposibilidad en algunos casos de anticiparlos, identificar su zona de impacto, prever su magnitud y proyectar sus secuelas. Por lo tanto, los desastres naturales -como un evento físico- pasan a ser desastres sociales cuando afectan a una población y se convierten en desastres sociales complejos debido al peligro y capacidad que tienen las personas para enfrentarlos (Campos-Vargas et al., 2015). Aún más, cuando los desastres golpean fuertemente áreas pobladas, el costo en vidas 
humanas, infraestructura y actividades económicas pueden ser devastadoras, pudiendo obligar al desplazamiento y posterior reasentamiento de la población (Naik et al., 2007).

La discusión en torno al desplazamiento como fenómeno socio-espacial ha llevado al surgimiento de una serie de categorías que intentan abordar este fenómeno, y particularmente de quienes han sido denominados desplazados ambientales, migrantes por causas ambientales, desplazados del desarrollo, refugiados ambientales, entre otras categorías (Reuveny y Peterson, 2007; Arenas, 2007; Seijo, 2007; Chafe, 2007), pero que poseen un factor común: los problemas del entorno o el medio ambiente, e incluso el cambio climático, como una de las causas directas que generan el desplazamiento y que obligan al reasentamiento de la población. Esto nos obliga a reconocer un debate inconcluso en torno a la conceptualización de los desplazados y -particularmente- de los desplazados por causas ambientales, el cual hasta hoy no ha sido resuelto.

Si bien dicha concepción es un avance, la situación para aquellas personas que se han visto obligadas a dejar su hogar tiene implícito el componente "peligro e inmediatez", asociado, tanto a factores de origen natural, como antrópicos. Sin embargo, tal noción deja fuera un número considerable de situaciones donde otros factores como la erosión, deforestación, procesos de remoción en masa, falta de recursos hídricos, o cualquier otro fenómeno físico, derivado incluso del calentamiento global, podrían obligar a una comunidad a dejar su lugar de origen.

Como hasta aquí se ha mencionado, existen diferentes enfoques que permiten clasificar y dar cuenta de la situación en que se encuentra un número importante de personas vinculadas al fenómeno del desplazamiento. Si bien es posible encontrar matices en torno a lo que constituye, por ejemplo un migrante económico, laboral, desplazado o refugiado; existe por otro lado un debate en torno al cual no hay consenso y dónde los diferentes actores involucrados no han logrado definir una posición única. Esto se daría particularmente con el surgimiento de otras categorías que refieren a fenómenos que definen a la población afectada como: desplazados ambientales, refugiados ambientales, migrantes forzados por causas ambientales, dependiendo de cómo sea considerado, y particularmente el respaldo o protección ante la situación que estas personas se encuentran. 
Ahora bien, es importante reconocer que desde el mundo académico hay un esfuerzo por reconocer y posicionar a los desplazados por factores ambientales; no es posible afirmar lo mismo para los organismos internacionales. Estos no han reconocido como tal a la población en dichas condiciones, ni tampoco lo han hecho las normas internacionales dirigidas a la protección de los desplazados (Borrás, 2006). Por extensión, es posible desprender que no existe un sistema integrado de protección, que resguarde la vida de las personas que se encuentran en tal condición.

Una de las principales dificultades en el último tiempo ha sido identificar o etiquetar a los migrantes medioambientales. Si bien es posible reconocer una excepción para aquellos casos donde pueblos completos se han visto obligados al desplazamiento a raíz de un desastre natural, es aún complejo establecer el vínculo con variables relacionadas al cambio climático o la degradación del medio ambiente, las que actúan en combinación con otros factores como los socioeconómicos y políticos, que condicionan la salida de las personas desde su lugar de origen. Boncour (2009) plantea que son estas las causas por las que los migrantes medioambientales no poseen un estatus claramente definido.

Transversalmente a las categorías antes señaladas, la noción del desplazamiento tiene como elemento constituyente el arraigo y desarraigo. La localidad constituye el hábitat donde las personas se encuentran arraigadas en base a lazos familiares, donde existe un vecindario y donde el asentamiento es un lugar construido por ellos. Allí surgen historias individuales y colectivas, se arman redes sociales, laborales, comunitarias y de amistad.

Cuando existe un desplazamiento y un reasentamiento, las personas son ubicadas en un lugar ajeno, que no es propio, ni elegido por ellos y por lo tanto que no genera arraigo ni identidad; a este lugar llegan con lo poco que tienen, con lo que les queda. (Rojas, 2010). Si consideramos un lugar como identidad, relacional e histórica, un espacio que no puede definirse ni como espacio de identidad ni como relacional ni histórico, definirá un no lugar, es decir, que no son en sí espacios antropológicos (Augé, 2000, p. 83).

Asimismo, cuando hablamos de desplazamiento, dos conceptos adquieren particular sentido. En primer lugar, la desterritorialización que reconoce los procesos de movilización caracterizados por altas condiciones de deprivación; entendida como una movilidad desigual, esta se intensifica en los grupos más precarizados, en espacios inestables e 
inseguros produciendo en las personas una pérdida de referencias simbólico-territoriales (Haesbaert, 2013, p.33). De esta manera, los habitantes de zonas altamente expuestas a amenazas naturales y que se han visto obligados a desplazarse debido a un desastre natural, son grupos que han tenido un escaso control sobre su territorio producto de sus precarias condiciones de vida. En segundo lugar, el proceso de reasentamiento que se da en un lugar completamente diferente al de origen, en cuanto al sistema de relaciones sociales y territoriales, implica que a partir de la desterritorialización previa, nuevos mecanismos de reterritorialización, en palabras de Haesbaert (2013), se desarrollarán como producto de las dinámicas y relaciones sociales emergentes en el nuevo asentamiento. Por lo tanto, resultará importante reconocer cómo la desterritorialización y reterritorialización, entro otros aspectos, buscan hacer hincapié en identificar las condiciones de precarización propias de un contexto de vulneración social que se dan en los procesos de desplazamiento.

\subsection{Vulnerabilidad social de desplazados}

Considerando los antecedentes antes expuestos, una de las perspectivas que cobra mayor importancia, que se ajusta de mejor manera al fenómeno analizado y que nos entregará las principales orientaciones a lo largo de este trabajo, corresponde a la categoría de desplazados internos. Entenderemos por ello a personas o un grupo de personas que han sido forzadas u obligadas a huir o a dejar su casa o lugar de residencia habitual, en particular como resultado de, o con el fin de evitar los efectos de, conflicto armado, situaciones de violencia generalizada, violaciones de derechos humanos, o desastres naturales o provocados por el hombre, y que no han cruzado una frontera internacionalmente reconocida (OIM, 1998).

Cabe señalar que la salida no implica necesariamente traspasar un límite administrativo, independiente si éste es comunal, provincial o regional, sino más bien, corresponde al reconocimiento de la situación que enfrentan las personas, en función del fenómeno que los obliga a dejar su hogar. Es por ello que la OIM señala algunas consideraciones en torno al fenómeno del desplazamiento, reconociendo que las personas desplazadas en el interior no corresponden a una definición legal, sino más bien descriptiva y que tienen necesidades especiales producto de su desplazamiento (Ibíd., p. 5). Esto sería particularmente 
importante cuando las personas están en campamentos y han desaparecido sus barrios, pero no para dar un trato especial, sino para prestar atención dada las difíciles necesidades que arrastran. Por tanto, se evidencian condiciones de desarraigo, separación de las redes de apoyo, despojo de recursos y que el desplazamiento como tal expone a sus víctimas a riesgos y vulnerabilidades adicionales.

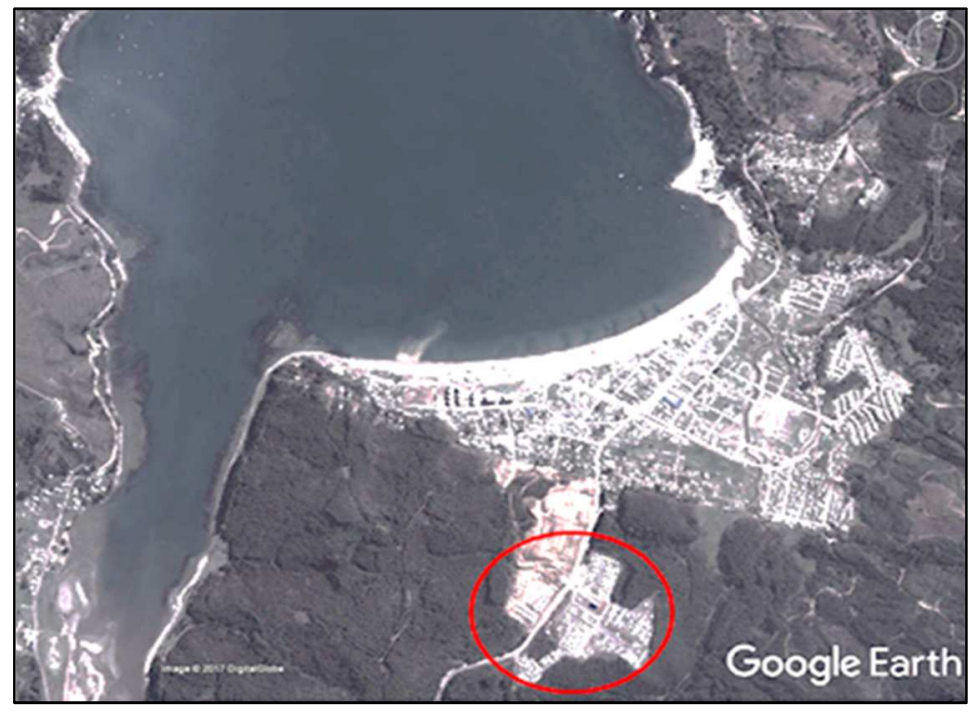

Figura 2 - Localidad de Dichato y reasentamiento de emergencia (Fuente: Google Earth; captura del 26 de agosto de 2012).

La Figura 2 muestra el reasentamiento de emergencia que albergó a más de 1.800 personas que vivían en la zona destruida por el terremoto y tsunami y que obligó la construcción de viviendas temporales ubicadas en la parte alta de esta localidad durante el proceso de reconstrucción. De esta manera, el reasentamiento se constituyó en el campamento de emergencia más grande de Chile generado a raíz del terremoto y tsunami de 2010.

Por lo tanto, la condición de desplazados, está dada por el desencadenamiento de un desastre que genera la destrucción del lugar de origen, la pérdida de bienes materiales y el valor simbólico de ellos, junto con la destrucción del tejido social que obliga a un reasentamiento temporal en condiciones de alta complejidad. En este contexto, se desconoce la disponibilidad de activos de los hogares y las estrategias de movilización de tales recursos para enfrentar un contexto post-desastre.

Si consideramos la clasificación de activos de Kaztman (1999) en: físicos, financieros, humanos y sociales, y entendemos los activos como insumos básicos que permiten el desarrollo de cualquier persona en una sociedad; la población afectada por un desastre 
natural y obligada al desplazamiento, se encontraría en un contexto particular de vulnerabilidad y con la necesidad de articular nuevas estrategias de movilización de dichos recursos. De esta manera resulta de particular interés, comprender cuáles fueron los activos disponibles que adquieren mayor importancia y de qué manera los hogares desarrollaron estrategias para hacer frente a la situación de vulneración que enfrentaron.

\section{Consideraciones metodológicas}

Acorde a los objetivos definidos para este trabajo, se elaboró un diseño mixto, donde se integran diferentes técnicas de investigación, tanto cualitativas como cuantitativas. De esto se desprende un diseño que involucra un proceso de combinación de métodos donde se trata de integrar subsidiariamente un método a otro, en este caso, el método cualitativo al método cuantitativo, para así fortalecer la validez de este último (Bericat, 1998).

La primera etapa de carácter exploratorio, consideró el desarrollo de entrevistas a 14 jefes de hogar que fueron analizadas considerando elementos de la Teoría Fundamentada de Glasser y Strauss (1967). Así se pudo conocer la vida cotidiana de las personas en condición de desplazamiento, permitiendo identificar las estrategias de movilización de activos implementadas.

La segunda etapa de carácter relacional, consideró el desarrollo de un cuestionario de activos de vulnerabilidad aplicado a 179 hogares, sobre un total de 453 hogares desplazados, ${ }^{1}$ número óptimo para lograr un nivel de representatividad adecuado de la población antes descrita, considerando un margen de error del 5\% y un nivel de confianza superior al $90 \% .^{2}$ Dicho cuestionario caracterizó a los hogares en función de 20 variables que para este contexto específico adquieren importancia para la determinación de la

\footnotetext{
${ }^{1}$ El total de familias desplazadas que forman los reasentamientos de emergencia corresponde a 453 familias (1.800 personas aprox.) en la localidad de Dichato, según datos del Ministerio de Vivienda y Urbanismo.

${ }^{2}$ Cabe señalar que el nivel de confianza que se esperaba lograr en esta investigación era de un 95\%; sin embargo, la muestra de 179 casos (que representa un 91\% de confianza e igualmente significativo) fue el máximo de hogares al que se logró acceder, considerando que algunos jefes de hogar fueron reticentes a proporcionar información referente al tema analizado, dada la complejidad propia de este contexto.
} 
vulnerabilidad social ${ }^{3}$ : hacinamiento, condición vivienda de emergencia, suministro de agua potable, servicio higiénico, vehículos, terrenos, ingreso autónomo, capacidad de ahorro, iniciativa comercial, participación en organizaciones sociales, red de apoyo familiar, nivel educacional, capacitaciones, tipo de empleo, tipo de contrato laboral, incremento de ingresos, restricción de consumos, salud familiar, enfermedad y situación de discapacidad.

Los datos obtenidos permitieron realizar un análisis de estadística descriptiva y un análisis factorial de correspondencias para la construcción del índice, proceso que fue apoyado a través del software SPSSC versión 18. El análisis factorial de correspondencias como procedimiento central de este trabajo, es una técnica estadística que busca simplificar un conjunto de resultados que nos proporciona una matriz de correlaciones, identificando el posible agrupamiento de variables y modalidades, según el comportamiento que los sujetos manifiestan en determinadas características normalmente de una sola variable discreta. Con ello se buscan las interrelaciones entre varias modalidades de distintas variables de forma que pueda conocerse si algunas actúan de manera semejante y a la vez distinta de otro conjunto de ellas (Calvo, 1993). De esta manera, permite identificar los componentes que agrupan las variables homogéneas de vulnerabilidad, pero de diferentes factores, con el objetivo de determinar la existencia de dimensiones latentes de vulnerabilidad social.

\section{Resultados y discusión}

Las 14 entrevistas realizadas a jefes de hogar, permitieron identificar el portafolio de activos de vulnerabilidad que disponen los hogares, conocer las características propias del contexto post-desastre y las estrategias de movilización de activos utilizadas por los hogares desplazados.

Respecto de los activos físicos, los recursos que adquieren importancia están asociados a los de mayor avalúo monetario, tales como: terrenos y vivienda de origen, vehículos y vivienda de emergencia. Los elementos propios de un contexto post-desastre que condicionan tanto los activos de vivienda y vehículos, se asocian a la destrucción total o parcial de estos

\footnotetext{
${ }^{3}$ Parte importante de estas variables se definieron en función de la etapa exploratoria y el desarrollo de las entrevistas a los jefes de hogares desplazados.
} 
recursos. En el caso de los terrenos, estos están fuertemente ligados a la incertidumbre respecto del uso de las propiedades, las cuales son sujeto de una eventual expropiación para el futuro proceso de reconstrucción de la localidad. Las viviendas de emergencia, se caracterizan por sus condiciones de hacinamiento y altísima precariedad (la vivienda de emergencia considera una habitación única para dormitorio, cocina y estar), además de encontrarse en un asentamiento que carece de servicios básicos (solo se cuenta con agua potable y servicios sanitarios comunes) reflejando condiciones poco dignas para la estadía de las personas. Las estrategias de movilización de activos se concentran fundamentalmente en generar mecanismos para mejorar la vivienda de emergencia. Es decir, los esfuerzos para enfrentar un contexto de vulneración pasa por ocupar dicho activo como un mecanismo que permita mejorar las condiciones de vida a través de: i) la ampliación o habilitación de nuevas habitaciones en la vivienda de emergencia; ii) habilitación informal de agua potable al interior de la vivienda y; iii) habilitación de servicio sanitario propio.

Los activos económicos que adquieren relevancia en esta etapa corresponden a los ingresos del hogar, ahorros e iniciativas comerciales. Los elementos de contexto que condicionan los ingresos están dados por la pérdida de fuentes laborales, destrucción de la actividad turística, destrucción de la actividad pesquera y destrucción de servicios menores en el borde costero y plano de Dichato. Los últimos tres elementos condicionan igualmente activos asociados al desarrollo de iniciativas comerciales. En el caso de los ahorros, está claramente limitado a los niveles de ingreso del hogar, los que también se ven mermados luego del terremoto y tsunami de 2010. Las estrategias de movilización de recursos para activos económicos corresponden a: i) desarrollo de iniciativas comerciales de carácter formal y; ii) desarrollo de iniciativas comerciales informales, ambas con el objeto de buscar una posibilidad para incrementar los ingresos del hogar.

Los activos de capital social se componen de la red de apoyo familiar y una red social externa al grupo familiar. La primera red enfrenta como elementos de contexto, la desestabilización de la misma; la existencia de hogares que conforman la red y que se encuentran en iguales condiciones de vulneración de los hogares analizados y; hogares de la red familiar que no hayan sido afectados por el desastre en los cuales se concentra el apoyo requerido. La red social (externa a la familia) está condicionada por la destrucción de la red comunitaria; el surgimiento de nuevos liderazgos y roles dirigenciales (y paralelamente el 
agotamiento de estos últimos) y; la intervención de ONG que promueven la articulación comunitaria post-desastre. En el caso de las estrategias de movilización de activos, son relevadas por parte de los hogares el fortalecimiento y apoyo de la red familiar como elemento prioritario.

La batería de activos de capital humano está compuesta por el nivel educacional del jefe de hogar, trabajo, relaciones del hogar y salud familiar. El nivel educacional por sí mismo no se ve afectado en un contexto post-desastre, más bien se constituye en un elemento diferenciador, que permite acceder a un tipo de empleo acorde a los años de estudios obtenidos. El acceso a un determinado tipo de trabajo, se encuentra afectado por la pérdida de fuentes laborales y la destrucción de la actividad turística, pesquera y de servicios menores, al igual que el activo asociado a ingresos. Las relaciones del hogar, que responden a acuerdos de los miembros para enfrentar contextos de vulneración, inevitablemente implican una alteración y ajustes acorde a las condiciones de vida del hogar. La salud familiar, si bien se asocia a aspectos físicos y mentales, ésta última adquiere importancia en la medida que el desastre natural, el desplazamiento y el reasentamiento genera una inestabilidad emocional importante en los miembros del hogar.

Finalmente, las estrategias desarrolladas por los hogares se agrupan como activos de relaciones del hogar, a través de acuerdos de todos los miembros del hogar para: i) reducir gastos y ii) generar ingresos adicionales que permitan enfrentar de mejor manera un contexto de alta vulneración.

El esquema conceptual que se presenta a continuación, sintetiza los resultados de esta etapa de análisis: 


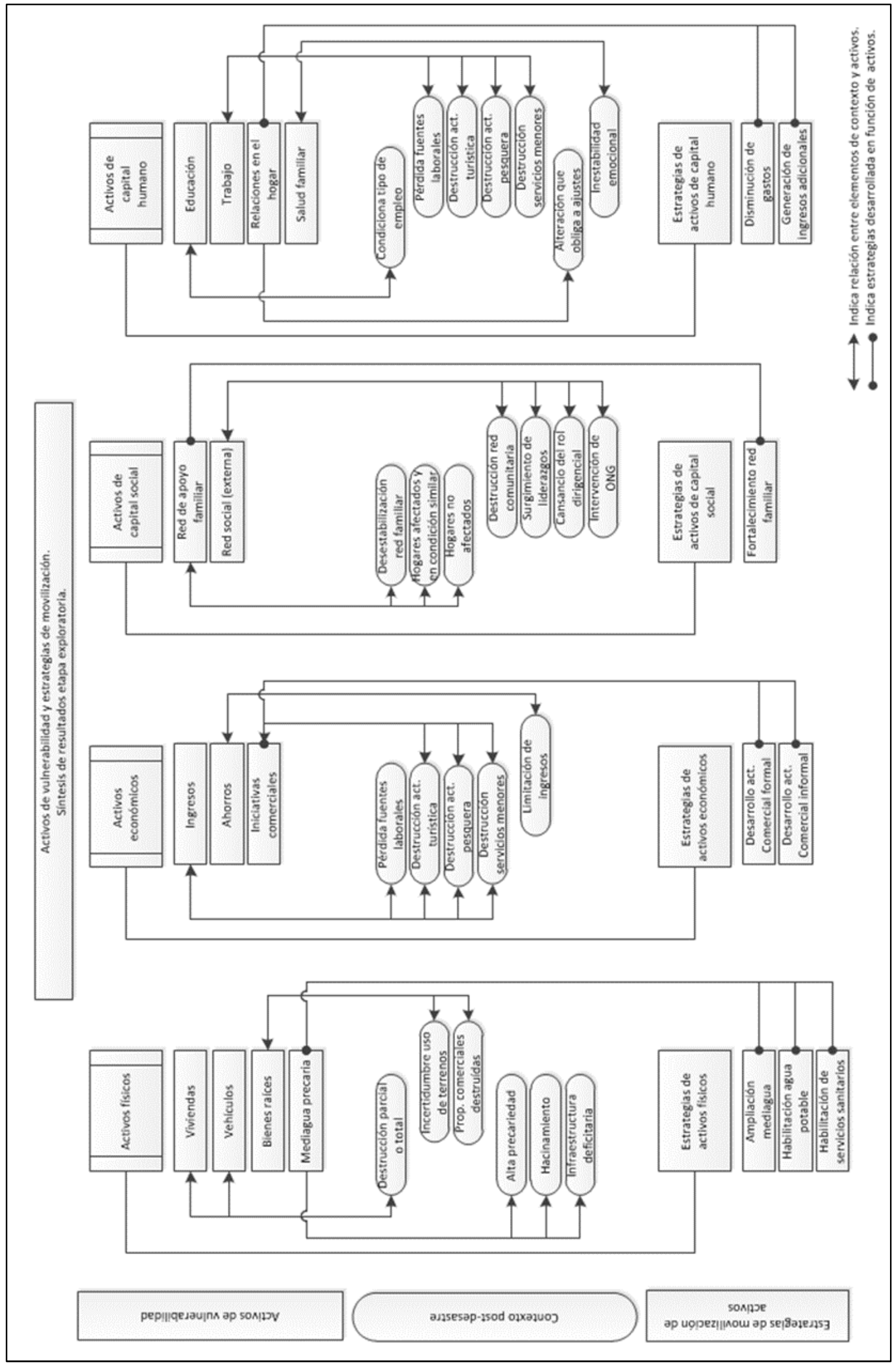

Figura 3 - Esquema conceptual de la etapa exploratoria (Fuente: Elaboración propia). 
Para la segunda etapa que consideró la construcción del índice de vulnerabilidad social de población desplazada, se encuestaron 179 hogares y se evaluó la batería de activos de vulnerabilidad disponibles, sus respectivas variables asociadas y las estrategias de movilización implementadas. Es importante señalar que las variables consideradas adquieren una valoración entre 0 y 1 dependiendo de la presencia total o parcial de activos. Por lo tanto, un valor igual a 0 representa menor vulnerabilidad y un valor igual a 1 implica mayor vulnerabilidad, con los respectivos gradientes que puedan existir. Por ejemplo, dentro de los activos físicos se encuentra la vivienda, siendo una de sus variables que la componen hacinamiento; un hogar que no se encuentra en condiciones de hacinamiento adquiere una valoración igual a 0 , mientras que un hogar con hacinamiento crítico adquiere una valoración igual a 1 . Considerando las 20 variables que componen el índice se obtiene la siguiente medida:

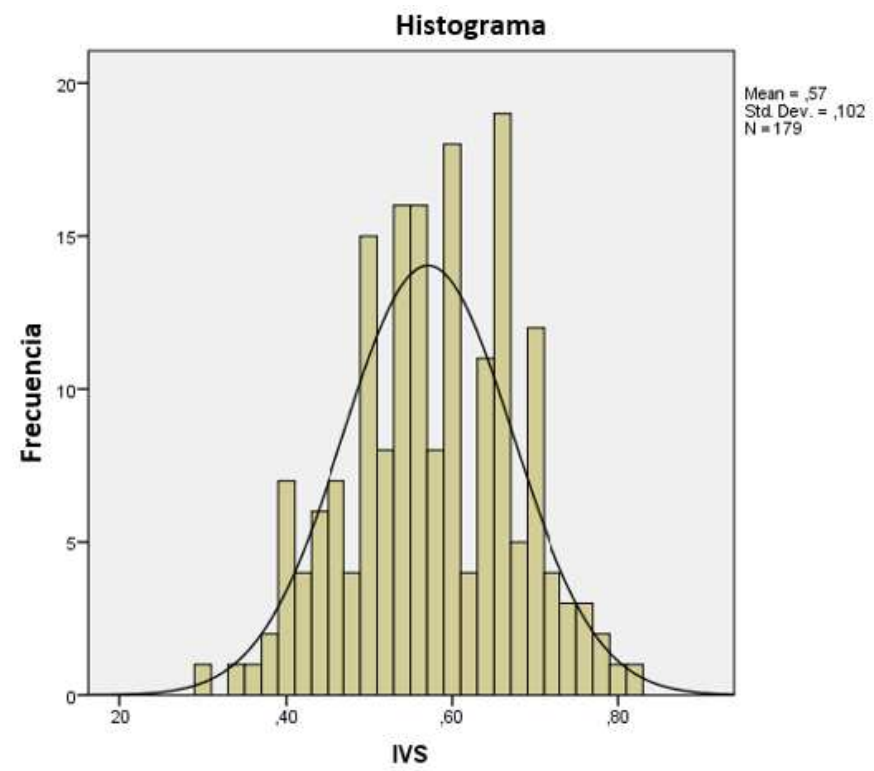

Figura 4 - Histograma Índice de vulnerabilidad social para hogares desplazados (Fuente: Elaboración propia).

Con base en lo anterior, se observa que la media de los hogares desplazados corresponde a 0,57 con una desviación estándar de 0,10.

De las 20 variables que componen el modelo que describe la totalidad de las dimensiones propuestas en la construcción de un índice de vulnerabilidad social para hogares desplazados, fue posible identificar 8 de ellas que presentan mayor correlación con el índice propuesto: educación, incremento de ingresos, vehículo propio, capacitación, iniciativa comercial, discapacidad, salud grupo familiar y enfermedad crónica. El factor de extracción, 
entendido como la proporción de la varianza que explicar el modelo factorial nos permite identificar la variable que más contribuye al análisis, este caso enfermedad crónica con un $54,6 \%$ de variabilidad y la que menor contribución realiza es capacitación, con un 34,5\%de variabilidad. Respecto de la prueba de Kaiser-Meyer Olkin (KMO), medida que determina si es viable el desarrollo de un análisis factorial arrojó una medida de 0,629 siendo ésta aceptable, considerando que una medida sobre 0,7 es considerada como alta. Asimismo, la prueba de esfericidad de Barlett, que busca determinar la homogeneidad de varianzas, es significativo con un valor de 0 y nos permite rechazar la hipótesis nula respecto de una matriz de identidad, ratificando la viabilidad o pertinencia de utilizar el análisis factorial propuesto.

Considerando que con este análisis se busca determinar cómo se agrupan determinadas variables en base de a una dimensión latente denominada factor, la varianza total explicada permite identificar el número de factores. Se obtuvieron dos autovalores observables mayores a 1,0. Entre ellos se representa un $43,577 \%$ de la varianza acumulada. De esta manera, el análisis puede definir dos factores que agruparían de manera importante la varianza de las ocho variables iniciales. Su representación gráfica, con el fin de facilitar la lectura quedaría de la siguiente manera:

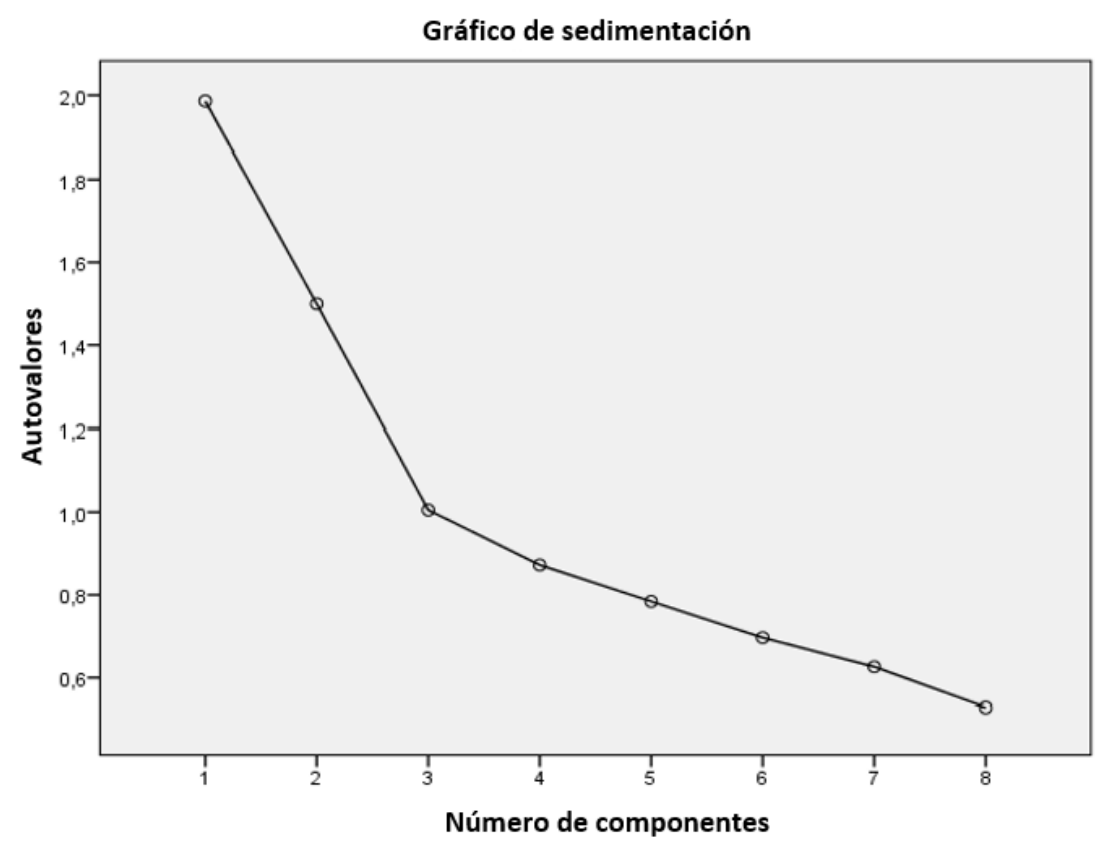

Figura 5 - Gráfico de sedimentación (Fuente: Elaboración propia). 
El gráfico de sedimentación permite identificar con claridad un punto de inflexión en torno a la varianza proporcionada por los autovalores. Dicha distinción, en este caso, genera 2 autovalores superiores a 1,0 y 5 autovalores que podrían ser considerados como residuales a partir del factor 3, dada su baja inclinación en contraste con la línea formada por los primeros dos factores. Por lo tanto, a partir del tercer factor, se pierde la pendiente pronunciada y generada por los factores 1 y 2, ratificando así la extracción de tales componentes.

De la solución factorial se desprendió que las variables que saturan el factor 1 son educación, salud del grupo familiar, discapacidad, capacitación, mientras que el factor 2 es saturado por incremento de ingresos, enfermedad crónica, iniciativa comercial y vehículo propio.

Definida ya la extracción de los factores, se optó por un proceso de rotación varimax, método de variación ortogonal que permitió minimizar el número de variables que logran una saturación importante para cada factor, en otras palabras, se logra una mayor correspondencia entre una variable y un factor. Esto se representa en un gráfico de factores en espacio factorial rotado:

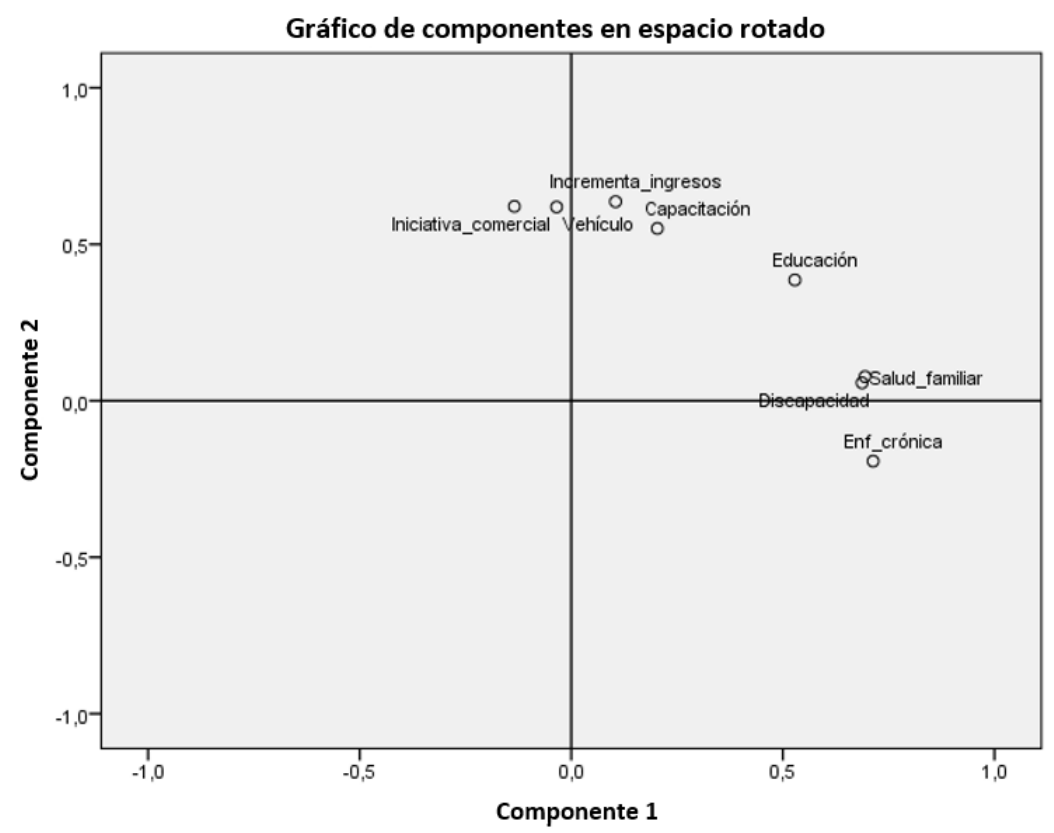

Figura 6 - Factores en espacio factorial rotado (Fuente: Elaboración propia).

El espacio factorial de este gráfico, donde cada una de las coordenadas descritas representa las saturaciones de las variables previamente señaladas, permite apreciar cómo se agrupan 
las variables en torno a los dos ejes o factores. Respecto del factor 1 , la variable enfermedad crónica $(0,713)$ posee una correlación alta y positiva; y las variables salud del grupo familiar $(0,694)$, discapacidad $(0,687)$ y educación $(0,528)$ poseen una correlación moderada y positiva. En el factor 2 las variables incremento de ingresos $(0,636)$, iniciativa comercial $(0,621)$, vehículo propio $(0,619)$ y capacitación $(0,551)$ poseen una correlación moderada y positiva.

Con base en lo anterior y considerando las características de las variables que componen el factor 1, lo denominaremos factores condicionantes de vulnerabilidad. En un contexto postdesastre, se puede advertir que tanto la educación del jefe de hogar, como las condiciones de salud del grupo familiar resultan claves al momento de enfrentar contextos de alta complejidad y vulnerabilidad. Probablemente, un bajo nivel educativo se asocia con trabajo precario y menores ingresos, mientras que la salud del grupo familiar, incluyendo la presencia de al menos un discapacitado físico o mental, se traduce en costos médicos y dependencia e incluso la merma de un individuo que puede aportar con ingresos al hogar para enfrentar las condiciones de vulneración propias de este contexto.

El factor 2 es denominado mecanismos de adaptación e involucra la movilización de algunos activos como la capacitación y el uso de vehículos propios con múltiples fines; como así también el desarrollo de iniciativas que fundamentalmente aluden a generar mayores ingresos económicos. Tales iniciativas pueden adquirir diferentes modalidades como por ejemplo, la incorporación a actividades laborales de jóvenes y mujeres, desarrollo de actividades informales como el comercio ambulante, venta informal de alimentos o prestaciones de servicios informales menores como costuras y arreglos de albañilería.

Es importante destacar que el surgimiento tanto de los factores condicionantes de vulnerabilidad, como de los mecanismos de adaptación, responden a un contexto específico de vulnerabilidad para la población desplazada de Dichato. Esto ha permitido comprender que dentro de la batería de activos, existen variables que dada su naturaleza, funcionan más bien como pasivos de vulnerabilidad desde la perspectiva de Kaztman (1999). Es decir, existen algunas variables que impiden o condicionan la capacidad de los hogares para movilizar o aprovechar los activos que poseen, situación que aparece con claridad cuando entre los integrantes hay personas con enfermedades crónicas, discapacitados, baja 
percepción del estado de salud familiar y cuando el nivel educativo del jefe de hogar es bajo.

Finalmente, los mecanismos de adaptación desarrollados por los hogares desplazados y reasentados en viviendas de emergencia de Dichato, implicaron que los integrantes de un hogar se organizaran y decidieran: generar mecanismos para aumentar sus ingresos, desarrollar alguna actividad comercial de carácter formal, potenciar el uso de un bien de alto valor como un vehículo (ya sea por venta o explotación comercial informal) o que los jefes de hogar se capacitaran con el fin de mejorar sus oportunidades laborales y por extensión sus condiciones de vida; todo esto en un contexto de reterritorialización condicionado por un proceso de desplazamiento y reasentamiento en un campamento de emergencia.

\section{Conclusiones}

Los antecedentes expuestos han permitido conocer diferentes aspectos de las condiciones de vulnerabilidad de los hogares desplazados, los niveles que esta alcanza, el surgimiento de dimensiones latentes respecto del comportamiento de las variables que la determinan y la identificación de las principales estrategias desarrolladas por los hogares acorde al objetivo propuesto. De lo anterior se puede concluir que:

i. El desplazamiento y la estadía en un asentamiento de emergencia, significó que los hogares reasentados enfrentaran una multiplicidad de problemas sociales y económicos que condicionaron y alteraron gravemente su calidad de vida, y por lo tanto se expusieron a mayores niveles de vulnerabilidad. Así, los principales factores de contexto y vulnerabilidad a los que se puede asociar la condición del desplazamiento, implican reconocer: a) la inexistencia de un arraigo mínimo con el asentamiento de emergencia; b) desarticulación de redes sociales existentes previo al desplazamiento y reasentamiento; c) percepción de aislamiento respecto del lugar de origen (plano de Dichato); d) lejanía respecto del borde costero; y e) alteraciones en las formas de accesibilidad al centro de la localidad. Si bien las condiciones por las que se releva la dimensión del desplazamiento pueden ser múltiples, los cinco puntos antes descritos resumen en buena medida el contexto en que este fenómeno 
se desarrolla.

ii. Respecto de las condiciones de vulneración de los hogares desplazados, el portafolio de activos de estos, representa un nivel de vulnerabilidad promedio de 0,57. Si bien esta medida no es totalmente comparable a otros contextos de vulnerabilidad, permite señalar que los hogares durante la estadía en los asentamientos de emergencia movilizan y desarrollan estrategias que los sitúan en un nivel inclinado hacia condiciones de mayor vulneración.

iii. Del total de variables que componen el índice propuesto, particularmente en un contexto condicionado por el desencadenamiento de un desastre y la situación de desplazamiento de los hogares analizados, podemos señalar que: salud familiar, condición de discapacidad, educación, incremento de ingresos, iniciativa comercial, vehículo propio y capacitación resultan claves como variables de distinción para determinar la vulnerabilidad acorde a sus respectivas modalidades o categorías.

iv. Considerando la búsqueda de dimensiones latentes en torno a las que se agrupan las variables correlacionadas con el índice propuesto se puede señalar que existen factores condicionantes de vulnerabilidad determinado por las variables: enfermedad crónica, salud del grupo familiar, discapacidad y educación que actúan como elementos que limitan la movilización de activos de los hogares analizados y el desarrollo de estrategias para enfrentar condiciones de vulneración en un contexto post-desastre. Asimismo, se identifican mecanismos de adaptación, determinados por las variables: incremento de ingresos, desarrollo de iniciativa comercial, vehículo propio y capacitación que dan cuenta de una situación donde los hogares que enfrentan mayores niveles de vulnerabilidad, se ven obligados a desarrollar una serie estrategias de ajuste o movilización de activos, que les permiten enfrentar de mejor manera las condiciones de vida en las que se encuentran.

v. No se puede concluir este trabajo sin reflexionar en torno a los múltiples desafíos que impone un fenómeno de estas características. Desde una perspectiva disciplinaria, se puede señalar que los mecanismos de adaptación utilizados en un contexto de vulneración social, permiten a la población desplazada y reasentada en campamentos de emergencia mejorar sus condiciones de vida. Sin embargo, también existen factores condicionantes de vulnerabilidad que limitan la capacidad de los hogares para enfrentar un contexto de alta 
complejidad por medio del uso y movilización de sus propios recursos. Es sobre dichos factores que resultará preponderante que los organismos del Estado trabajen focalizadamente para que las personas estén más preparadas y cuenten con herramientas que les permiten movilizar la mayor cantidad de activos que puedan disponer. De esta manera, los hogares desplazados podrán enfrentar de mejor forma las condiciones de vulneración en las que permanecen mientras dura el largo proceso de reconstrucción de su asentamiento de origen o, eventualmente, la construcción de un nuevo asentamiento definitivo.

\section{Referencias bibliográficas}

ÁLVAREZ, Guadalupe; TUÑON, Esperanza. Vulnerabilidad social de la población desplazada ambienta por las inundaciones de 2007 en Tabasco (México). Cuadernos de Geografía: Revista Colombiana de Geografía. 2016, vol $25 \mathrm{~N}^{\circ} 1$, p. $123-138$.

ARAÚJO, Raquel; ROSA, Teresa. Socio-environmental vulnerability and disaster risk reduction: the role of espírito santo state (Brazil). Revista Ambiente \& Sociedade. 2014, vol XVII, N 4, p. 117-132.

ARENAS, Nuria. La normativa jurídica aplicable a los desplazados por causas medioambientales. En MARTíNEZ, Joan; PUIG, Ignacia; MONJO, Anna; ORTEGA, Miguel. Ecología Política. Cuadernos de Debate Internacional $N^{\circ}$ 33. Madrid: Editorial Icaria, 2007, p. 69-71. ISBN: 1130-6378.

AUGÉ, Marc. Los no lugares, espacios del anonimato: una antropología sobre la modernidad. Barcelona: Editorial Gedisa, 2000. ISBN: 84-7432-459-9.

BARRENECHEA, Julieta; GENTILE, Elvira; GONZÁLEZ, Silvia; NATENZON, Claudia. Una propuesta metodológica para el estudio de la vulnerabilidad social en el marco de la teoría social del riesgo. IV Jornadas de Sociología de la Facultad de Ciencias Sociales UBA, Buenos Aires, 2000.

BERICAT, Eduardo. La integración de los métodos cuantitativo y cualitativo en la investigación social: Significado y Medida. Barcelona: Editorial Ariel, 1998. ISBN 843441693.

BLAIKIE, Piers; CANON, Terry; DAVIS, Lan; WISNER, Ben. Vulnerabilidad: El entorno social, político y económico de los desastres. Bogotá: Red de Estudios Sociales en Prevención de Desastres en América Latina, 1996. ISBN: 958-601-664-1.

BONCOUR, Philippe. La hora de la verdad - Adaptación al cambio climático. [Digital]. Revista Migraciones. Otoño 2009: Disponible en: http://publications.iom.int/system/files/pdf/migration_autumn_09_sp.pdf

BORRÁS, Susana. Refugiados ambintales: El nuevo desafío del derecho internacional del medio ambiente. Revista de Derecho. 2006, N² 2, vol. XIX, p. 85-108.

BUSSO, Gustavo. Migración interna, pobreza y desarrollo territorial en el cono sur de América Latina: Impactos sociodemográficos de la migración interna a nivel de Divisiones Administrativas Mayores en Argentina, Bolivia, Brasil y Chile. En Reunión de Expertos sobre Población y Pobreza en América Latina y el Caribe. Santiago, 14 y 15 de noviembre de 2016. ELADE - División de Población CEPAL.

CALVO, Félix. Técnicas estadísticas multivariantes. Bilbao: Universidad de Deusto, 1993. ISBN: 9788474852974. 
CAMPOS-VARGAS, Milagros; Toscana-Aparicio, Alejandra; Campos Juan. Riesgos socionaturales: vulnerabilidad socioeconómica, justicia ambiental y justicia espacial. Cuadernos de Geografía: Revista Colombiana de Geografía. 2015, vol 24, N², p. 53-69.

CEPAL. Terremoto en Chile. Una primera mirada al 10 de marzo de 2010. [Digital]. Publicación de las Naciones Unidas, 2010. Disponible en: http://www.cepal.org/noticias/paginas/4/35494/2010-193-Terremoto-Rev1.pdf

CHAFE, Zoë. Las crecientes amenazas de desastre y su potencial riesgo de desplazamientos de población. En MARTínEZ, Joan; PUIG, Ignacia; MONJO, Anna; ORTEGA, Miguel. Ecología Política. Cuadernos de Debate Internacional $N^{\circ} 33$. Madrid: Editorial Icaria, 2007, p.84-89. ISBN: 1130-6378.

CUTTER, Susan; BORUFF, Bryan; SHIRLEY, W.Lynn. Social vulnerabilty to environmental hazards. Social Science Quarterly. 2003, vol 84, N², p.243-261.

CUTTER, Susan; FINCH, Christina. Temporal and spatial changes in social vulnerability to natural hazards. PNAS. 2008, vol $15 \mathrm{~N}^{\circ} 7$, p.2.301-2.306.

FILGUEIRA, Carlos. Estructura de oportunidades y vulnerabilidad social. Aproximaciones conceptuales recientes. En Seminario Internacional: Las diferentes expresiones de la vulnerabilidad social en América Latina y el Caribe, Santiago, 20 y 21 de junio de 2001. CEPAL-CELADE, Santiago.

FILGUEIRA, Carlos; PERI, Andrés. América Latina: los rostros de la pobreza y sus causas determinantes. Serie Población y Desarrollo. CEPAL, 2004, N²54. ISBN: 92-1-322538-5.

GLASSER, Barney; STRAUSS Anselm. The discovery of grounded theory: strategies for qualitative research. New York: Aldine Publishong Company, 1967.

GOLOVANEVSKY, Laura. Vulnerabilidad y transmisión intergeneracional de la pobreza. Un abordaje cuantitativo para Argentina en el siglo XXI. Tesis doctoral de Facultad de Ciencias Económicas. Universidad de Buenos Aires, 2007.

HAESBAERT, Rogério. Del mito de la desterritorialización a la multiterritorialidad. Revista Cultura y representaciones sociales, 2013, vol 8, N¹5, p. 9-42.

INE. Informe cartográfico de daños terremoto y tsunami de 2010. [Digital] Publicación INE Bío-Bío, 2010. Disponible en: http://www.inebiobio.cl/noticia.aspx?opc=ShowNew\&id=112

KAZTMAN, Rubén (Coord). Activos y estructuras de oportunidades. Estudio sobre las raíces de la vulnerabilidad social en Uruguay. [Digital] CEPAL, 1999, Uruguay. Disponible en: http://www.cepal.org/cgibin/getProd.asp?xml=/publicaciones/xml/2/10772/P10772.xml

KAZTMAN, Rubén, WORMALD, Guillermo (Coordinadores) Trabajo y Ciudadanía. Los cambiantes rostros de la integración y exclusión social en cuatro áreas metropolitanas de América Latina. [Digital] Universidad Católica de Uruguay. Uruguay, $2002 . \quad$ Disponible en: http://ucu.edu.uy/sites/default/files/libros/pdf/trabajo ciudadania.pdf. ISBN: 9974-54-036-3.

KAZTMAN, Rubén. Notas sobre la medición de la vulnerabilidad social. [Digital] En $5^{\circ}$ Taller Regional del MECOVI; la medición de la pobreza, métodos y aplicaciones, México, junio de 2000. Disponible en: http://www.eclac.cl/deype/mecovi/docs/TALLER5/24.pdf

KAZTMAN, Rubén. Seducidos y abandonados: el aislamiento social de los pobres urbanos. Revista de la CEPAL. Comisión Económica para América Latina y el Caribe, 2001, N 75, p. 171-189.

MOSER, Caroline; MCILWAINE, Cathy; HOLLAND, Jeremy. Household Responses to Poverty and Vulnerability: Confronting Crisis in Angyalfold, Budapest, Hungary. World Bank Publications, 1997.

MOSER, Caroline. Confronting Crisis: Comparative study of household responses to poverty and vulnerability in four poor urban communities. [Digital] Environmentally Sustainable Development Studies and Monographs. 1996, Series World Bank $\mathrm{N}^{\circ}$ 7, Washington. Disponible en: http://documents.worldbank.org/curated/en/334951468336549231/pdf/154620PUBOREPL00000010Box200P UBLIC0.pdf. ISBN: 0-8213-3561-8.

MOSER, Caroline. The asset vulnerability framework: Reassing urban poverty. World Development, $\mathrm{N}^{\circ} 26.1998$ 
NAIK, Asmita; STIGTER, Elca; LACZKO, Frank. Migration, development and Natural Disasters: Insights from the Indian Ocean Tsunami. Migration Research OIM. 2007, Series N³0, Switzerlan. ISBN: 1607-338X.

OIM. Principios rectores del desplazamiento interno. [Digital] Consejo Económico y Social de Naciones Unidas.

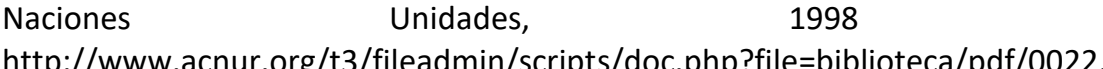

PIZARRO, Roberto. La vulnerabilidad social y sus desafíos: una mirada desde América Latina. Serie Estudios Estadísticos. CEPAL. 2001. ISBN: 1680-8770

REUVENY, Rafael; PETERSON, Ashley. Los refugiados ambientales y sus consecuencias en el futuro. En MARTínEZ, Joan., PUIG, Ignacia., MONJO, Anna., ORTEGA, Miguel. Ecología Política. Cuadernos de Debate Internacional $N^{\circ}$ 33. Madrid: Editorial Icaria, 2007, p.21-36. ISBN: 1130-6378.

ROJAS, Jorge. Vulnerabilidad social, neoliberalismo y desastre: sueños y temores de la comunidad desplazada/damnificada por el terremoto/tsunami. Revista Sociedad Hoy. Universidad de Concepción, 2010, $\mathrm{N}^{\circ} 19$, p. 113-140.

SANDOVAL-AYALA, Norma; SOARES-MORAES, Denise. Vulnerabilidad y activos familiares frente a riesgos. Caso de estudio en Ixil, Yucatán. Revista LiminaR. Estudios Sociales y Humanísticos. 2015, vol XII, N 1, p. 56-68.

SEIJO, Francisco. iModernización o emigración!: desplazados ambientales y repoblación forestal en Galicia desde 1950. En MARTíNEZ, Joan; PUIG, Ignacia; MONJO, Anna; ORTEGA, Miguel. Ecología Política. Cuadernos de Debate Internacional N 33. Madrid: Editorial Icaria, 2007, p. 69-71. ISBN: 1130-6378. 